\title{
The Impact of the Combination between Task Designs and Think-Aloud Approaches on Website Evaluation
}

\author{
Roobaea AlRoobaea ${ }^{1}$, Ali H. Al-Badi ${ }^{2}$ and Pam J. Mayhew ${ }^{1}$ \\ ${ }^{1}$ College of Computers and Information Systems, Taif University, Saudi Arabia and \\ School of Computing Sciences, University of East Anglia, Norwich, UK \\ ${ }^{2}$ Department of Information Systems, Sultan Qaboos University, Oman
}

Correspondence should be addressed to: Roobaea AlRoobaea; r.alrobaea@uea.ac.uk

Received date: 15 April 2013; Accepted date: 12 August 2013; Published date: 19 September 2013

Academic Editor: Jia Rong

Copyright (C) 2013. Roobaea AlRoobaea, Ali H. Al-Badi and Pam J. Mayhew. Distributed under Creative Commons CC-BY 3.0

\begin{abstract}
The growth of the Internet and related technologies has enabled the development of a new breed of dynamic website and applications that are growing rapidly in use, which have had a significant impact on many businesses. Usability testing is a method widely used for measuring the usability of a system from the perspective of real users.

The main aim of this paper is to explore the efficiency of three types of usability testing method by discovering usability problems through a combination of three proposed task designs and three types of 'think-aloud' approach, and to compare the results of these methods with those of the traditional usability testing method that has been published separately. This is a missing area in usability testing. The quantitative and qualitative data are collected and analysed in order to assess the efficiency of the three combinations in terms of the usability problems encountered and their severity, and the success rate and user satisfaction of the websites being tested, through the feedback received. The research findings show clearly that the task scenarios and how they are designed and combined with different types of 'think-aloud' approaches can influence the usability testing results by discovering different types of usability problems, and can affect the results of usability measures, such as success rate, errors number and time spent.
\end{abstract}

Keywords: Specific task, Guess task, Free task, Concurrent, Retrospective, Constructive Interaction approaches 


\section{Introduction}

The growth of the Internet and new technologies has created new dynamic websites that are growing rapidly in use and are having a significant impact on many businesses. These dynamic websites increasingly being developed in the midst of the Internet revolution and ever-improving information technologies. For example, elearning websites are now essential for all universities that have a physical workplace. They have websites, and particularly those for e-learning, that have become an integral part of their businesses. The Internet revolution has even led to a large number of universities functioning solely online, without the need of a physical workplace. To keep pace with this development; some companies and organizations such as Intel® Education and the BBC seek to build free online learning websites that are oriented to world-class education at all educational levels. This development in lifelong learning has made the learners' intention to continue using e-learning an increasingly critical issue. Consequently, quality is considered crucial to education in general, and to elearning in particular. Web design is a key factor in determining the success of $\mathrm{e}$ learning websites, and the users should take priority in the designers' eyes because usability problems in educational websites can have serious ramifications over and above failing to meet the users' needs.

Usability is one of the most significant aspects affecting the user experience. The techniques for measuring the quality of users' experience have been classified under the heading of 'ergonomics' and 'ease-of-use', but more recently under the heading of 'usability' [Oztekin \& Uysal, 2010]. ISO defines usability as "the extent to which the product can be used by specified users to achieve specified goals with effectiveness, efficiency, and satisfaction in a specified context of use" [ISO 9241-11, 1998]. During the past decade a variety of Usability Evaluation Methods (UEMs) have been developed, and a number of research studies have compared the effectiveness of different UEMs [Jeffries et al., 1991; Alrobai et al., 2012; AlRoobaea et al., 2013; AlRoobaea et al., 2013; AlRoobaea et al., 2013; AlRoobaea et al., 2013; Ali et al., 2013; Ahmed et al., 2013]. UEMs can be categorized as analytic or empirical; analytic UEMs include techniques such as Heuristic Evaluation (HE), whereas empirical UEMs include a wide range of methods and procedures that are often referred to simply as 'usability (or user) testing' [Nielsen \& Molich, 1990; Lewis \& Polson, 1992].

Briefly, usability testing is labour-intensive, as it requires real users of the product to be examined whilst performing a set of tasks. These users need to be observed carefully by evaluators in order to record the usability problems encountered by them. Overall, the most important aim of usability testing is the compilation of a list of the usability problems encountered, which will then indicate the necessary changes to make in order to improve the product [Lawand \& Hvannberg, 2002].

The recent literature has raised a number of questions and issues in the usability testing field that deserve further research from various viewpoints. For example, many researchers such as Fang and Holsapple (2011), and AlShamari and Mayhew (2010) have investigated the impact of task types on user testing results. However, the impact of task formulation and combining it with different 'think-aloud' approaches is a missing area in usability testing. This paper aims to investigate the efficacy of using three different combinations of task formulation and 'think-aloud' approaches in usability testing. Three out of nine combinations were chosen and tested, which were 'specific task with constructive interaction think-aloud', 'guess task with concurrent think-aloud', and finally, 'free task with retrospective thinkaloud'. These were measured against the number of problems found, the users' performance and the overall user experience, as well as other usability measures. This paper is organized thus: Section 2 presents a brief literature review relating to this study; Section 3 describes the methodology and techniques adopted; Section 4 contains the data analysis; Section 5 presents a discussion and highlights interesting findings; and 
Section 6 presents the conclusion and suggestions for future work.

\section{Literature Review}

The concept of user experience has become central to interaction design; it defines how a product behaves and is used in the real world [Rubin \& Chisnell, 2008]. Garrett (2010) describes user experience as what happens to the users when they interact with the system in question. Experience varies from person to person and from product to product because of something calling 'usability'. Usability is a one of the core concepts to have emerged from the humancomputer interaction (HCI) field. The literature reveals that usability is not a single 'one-dimensional' property of a user interface; there are many usability attributes that should be taken into account and measured during experimental sessions. For example, whether or not it is easy to learn and to remember, efficient and subjectively pleasing to use, and whether or not there are errors. There are a variety of methods for measuring the usability of a system or product, and they can be divided into three categories: usability testing (below), usability inspection (e.g. Heuristic Evaluation), and inquiry methods (e.g. interviewing users). As mentioned before, the first is empirical and the other two are analytical.

Usability testing (or user testing) is the method addressed in this paper. This is considered to be the most important evaluation method for ensuring system quality, and particularly for websites, although it is claimed to be costly [Oztekin \& Uysal, 2010]. It requires participants to perform a set of tasks, usually under laboratory conditions. These tasks are performed without including any information or clues as to how to complete the tasks or scenarios, and with no help provided to the user during the test session. The performance of these tasks are monitored through 'observation' and then assessed by the evaluator/researcher in order to record the usability problems encountered by the users. All the observed data, such as error numbers, time spent, success rate and user satisfaction, are recorded for analysis [Nielsen, 1994]. Dumas and Redish (1991) point out that a fruitful usability testing session requires careful planning and attention to detail. Thus, there is a general procedure for conducting user testing: 1) Planning a usability test; 2) Selecting a representative sample and recruiting participants; 3) Preparing the test materials and actual test environment; 4) Conducting the usability test; 5) Debriefing the participants; 6) Analysing the data of the usability test; and 7) Reporting the results and making recommendations to improve the design and effectiveness of the system or product. The aim of applying usability testing is to procure a list of usability problems, which are defined as flaws in the design of a system that make the attainment of a particular goal (through the use of the system) ineffective and/or inefficient, and thus lower the users' level of satisfaction in using it [Lewis \& Polson, 1992]. These problems can be classified into different groups, in which a numeric scale from 0 to 4 is often used to measure the severity of each problem. 0 means "not a usability problem at all", 1 means "cosmetic problem that should be fixed if enough time is available", 2 means "minor problem with low priority", 3 means "major problem with high priority", and 4 means "catastrophic problem that is imperative to fix". Usability testing involves the collection of measurable data that can be compared with those of other websites; in particular, data on time spent, task success rate, error numbers, satisfaction rate and other metrics [Tan et al., 2009].

There are various factors affecting usability testing and its results, as Figure 1 shows. These factors include the number of users ('sample size'), the usability measures employed, the users' characteristics ('user profiling'), the use of the 'think-aloud' protocol (in its varying forms), the formulation of the tasks, and environment testing. Firstly, usability testing requires real users to perform realistic tasks; however, there is no agreement amongst researchers about the most appropriate number of users. Nielsen (2000) claims that five users are enough to reveal $85 \%$ of all the usability problems in a user interface, and that time and resources are wasted if more than five 
are involved. Rubin and Chisnell (2008) confirm that the first of the three to five users can detect the majority of the usability problems, and that each additional user is unlikely to uncover new ones. Several researchers have suggested that 5 to 12 users are sufficient for the purposes of website usability testing; however, others have suggested that 20 users, for the purpose of benchmarking, are needed for an experiment [Nielsen, 2006; Rubin \& Chisnell, 2008]. Lindgarrd and Chattatichart (2007) confirm, in the findings of their paper which discusses user numbers and tasks, that "the result casts doubt on the role of the number of users in a usability test but confirms the important role of task coverage".

The second factor is 'usability measures'. This is the most important factor because any failure in measuring the level of usability means failure in the whole evaluation. There are three main ISO standards for measuring usability, and these are efficiency, effectiveness and user satisfaction. Efficiency can be calculated through measuring input rate, task completion time and other factors. Effectiveness can be worked out through measuring binary task completion, accuracy and the number of errors. Satisfaction can be measured by asking about the user's feelings towards the system or through conducting standardized questionnaires with the users after the test [Sauro \& Kindlund, 2005; Hornbaek, 2006]. However, there are other usability measures such as success rate, which is defined by Nielsen (2001) as the percentage of tasks that users complete correctly and successfully. He divides task completion into three categories, which are 'completed successfully without error', 'completed partially with some errors but with recovery from those errors', and 'failure to complete' [Nielsen, 2001]. Overall, usability measures can produce different types of result, but which system to measure, what to measure and how to measure it are critical questions in determining the most appropriate usability measures.

The third factor is 'user characteristics'; it is important to consider the user profile from all angles. Users differ in age, nationality, background, gender and, crucially, computing skills. Each one of these factors may play a significant role in usability testing. Molich et al., (2004) states, "usability testing effectiveness is dependent on the tasks given, the methodology used and the users' characteristics".

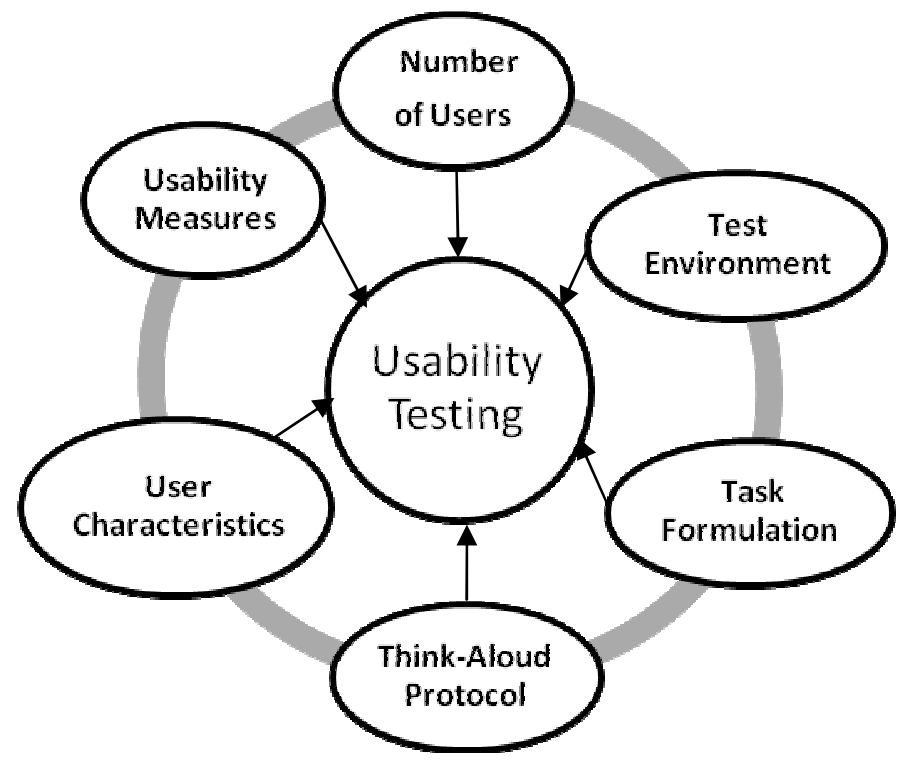

Figure 1: Factors that impact on the results of usability testing 
Regarding the fourth factor, there are various techniques that supplement usability testing, the 'think-aloud' protocol being the most widely used. It is employed during the test when the users are asked to think out loud whilst performing their tasks and their thoughts, feelings, and opinions are recorded. This technique can effectively help evaluators to capture how users interact with an interface and what is happening on the screen [Rubin \& Chisnell, 2008]. It has been claimed that one-third of 'severe' usability problems can be discovered through this technique [Ebling \& John, 2000]. However, the environmental setting of the usability test can sometimes influence the effectiveness of the 'think-aloud' protocol, and it does not always help when the users are not in their natural surroundings; this means that users may not feel as relaxed and may feel unable to talk or express their thoughts and ideas freely in a restricted and unfamiliar laboratory environment [Haak \& Jong, 2004]. Furthermore, Rubin and Chisnell (2008) suggested that if the tasks are designed to assess the efficiency of a system (i.e. measuring time spent on tasks) then 'think-aloud' protocol should be avoided as it may negatively impact on the performance of the users. The 'think-aloud' protocol has been generally used in achieving three goals; firstly, to find evidence for models and theories of cognitive processes; secondly, to discover and understand general patterns of behaviour in the interaction with documents or applications in order to create a scientific basis for designing; and thirdly, to test specific new documents or applications in order to troubleshoot and revise them [Krahmer \& Ummelen, 2004].

There are three types of 'think-aloud' protocol, which are 'concurrent', 'retrospective' and 'constructive interaction'. The concurrent 'think-aloud' type is the most common; this involves participants verbalising their thoughts whilst performing tasks in order to evaluate a product. 'Retrospective think-aloud' is less frequently used; in this method participants perform their tasks silently, and comment on their work afterwards by means of recording of their performance. Constructive interaction is more commonly known as 'co-discovery learning', where two participants work together in performing their tasks, verbalising their thoughts through interaction (with each other?) [Van den Haak et al., 2004]. On the one hand it has been argued that 'think-aloud' protocol should be avoided in certain circumstances, as mentioned above, but on the other hand, Tullis and Albert (2008) assess the degree to which it can actually influence users' performance, concluding that this technique can in fact enhance performance because it helps users to focus more on the tasks. However, some researchers when employing the concurrent type have concerns about reactivity, i.e. the possibility that the act of speaking concurrently may influence user performance through distracting their attention and concentration; the effort of fully verbalizing the steps taken in the task may change the ways that users attend to the task components. For this reason the retrospective 'think-aloud' type has been proposed in order to avoid the problems of 'concurrent think-aloud; it is assumed to be the most fruitful in terms of problems reported per participant [Van den Haak et al., 2004]. Furthermore, 'co-discovery learning' (constructive interaction) has been claimed to be the most suitable method for evaluating collaborative systems and also to be the most appropriate method for usability testing with children [Nielsen, 1994].

The fifth factor in usability testing is the design of the tasks. Many researchers are aware that task design is an important factor in the design of adequate Web usability tests. The tasks designed for Web usability testing should focus on the main functions of the system. The tasks should cover the following aspects: 1) Product page; 2) Category page; 3) Display of records; 4) Searching features; 5) Interactivity and participation features; and 6) Sorting and refining features. The tasks can be selected from four different perspectives. These are: 1) Tasks that are expected to detect usability problems; 2) Tasks that are based on the developer's experience; 3) Tasks that are designed for specific criteria; and 4) Tasks that are normally performed on the system. Researchers also recommend that the tasks should be short and clear, in the users' language, and based on the system's goals. 
Alshamari and Mayhew (2008) found that task design can play a vital role in the usability testing results, where it can be shown that changing the design of the task can produce differences in the results. They propose and examine three different types of task formulations, which are structured tasks, uncertain tasks and problem-solving tasks. They find that structured tasks are able to identify superficial and minor usability problems whereas the problemsolving tasks are able to identify usability disasters and major problems. Furthermore, Fang and Holsapple (2011), having designed two sets of tasks, which were 'simple tasks' and 'complex tasks', find that simple tasks impact differently on the website navigation structure, user performance, and perceptions of usability than complex tasks.

The sixth factor is the test environment. Generally, user testing takes place in a controlled laboratory. Tullis et al., (2002) discover several cases where the product works fine in the laboratory but not in the real world. They find that the conditions under which the product's use is tested are different to the conditions for actual use. Dix et al., (2004) list four aspects when seeking to understand why a laboratory experiment sometimes fails: 1) The users' motivation can be greatly diminished or even destroyed by the atmosphere of a controlled laboratory; 2) A laboratory does not take into account the social context (of supporting and motivating the users if they need it); 3) A laboratory setting does not consider the time context where, in reality, users may leave their work and resume it later; and 4) A laboratory does not take into account the user's work context (users may feel disinclined to invest time and effort in something that they see as someone else's job). However, conducting experiments in a laboratory can increase their validity, can facilitate system comparisons, and can offer a controlled area where all interactions with the system can be closely recorded and monitored [Lazar et al., 2010; Nielsen, 2009].

In conclusion, further research should be carried out to investigate the impact of task formulation on usability testing, as recommended by Fang and Holsapple (2011) and Alshamari and Mayhew (2008).
Furthermore, there is no research that investigates the impact on the results of user testing of a combination of different types of task formulation and different types of thinkaloud technique. Many researchers use traditional user testing which consist of simple tasks and observation [Dale et al., 2012; Manzari and Trinidad, 2013]. This research will also compare the proposed combined methods here with the results of the traditional user testing in the paper written by AlRoobaea et al., (2013) on the same websites. This will help us discover which method can provide a better performance in the identification of usability problems. Consequently, this research will consider all these gaps and it will present useful information in the HCI field.

\section{Research Methodology}

The experimental approach was selected to achieve the research objectives outlined above. The educational domain was chosen, and three educational websites were selected for different reasons; firstly, they are all completely free educational websites; secondly, they are interactive and are supported by three well-known companies with a wide range of users; and thirdly, they meet the research objectives and requirements through having various functions, processes and features. These websites are BBC KS3bitesize, Skoool, and Academic Earth. Three experiments were employed on each. Each experiment employed one design of task and one type of 'think-aloud' protocol. This section presents an explanation of the test procedures in these experiments, including the quantitative and qualitative data.

\section{Testing procedures}

The user testing was conducting by giving a quick introduction to the task designs; thinkaloud approaches and the purpose of the study to each user. The next step was to explain the testing environment and how to use the equipment, followed by a quick demonstration of how to think aloud for each test' while performing the given tasks. Prior to the tests the users had to read and sign the consent letter and fill in a user sheet that included his/her demographic data, 
such as computer skills, nationality and gender. All the above steps took approximately ten minutes for each test session. The timing of each test started when the user was given the task scenario sheet and asked to read and then perform the task. Once they had finished the session they were asked to rate their satisfaction levels. This was followed by a brief discussion.

\section{The Proposed Tasks}

The literature suggests that further work on task design is needed [Lindgaard \& Chattratichart, 2007; Alshamari \& Mayhew, 2008]. This is because giving users only one task design may not achieve the testing goals; however, offering users different designs of task may reveal more usability problems. Three designs were proposed for the tasks to be employed in this study. These three types were:

1. Specific Task: this design of task will guide the users to finding specific information relating to a number of specific questions. This task involves constructive interaction 'think-aloud' type (co-discovery learning), where two users work together in performing their tasks. This is designed to reveal potential usability problems. For example, the following task is to measure the usability of a website's registration process.

- Work with your friend and go to the website and register.

- Work with your friend and fill in the fields, following the instructions given.

2. Guess Task: in this task design the users should guess aloud, saying what information they expect to find behind the functions targeted in the test. This design of task relies on the fact that users are usually uncertain as to whether they will find the information that they are looking for whilst surfing a website. The task involves the concurrent 'think-aloud' type, which is the most commonly used. The following is an example of this task, which is to measure the usability of a website's post-a-question facility.

\section{- Guess how you can post your question.}

3. Free Task: in this task users are allowed to explore the target website freely, based on their own interests. This task involves the retrospective 'think-aloud' type, which means that users perform their tasks silently, and afterwards comment on their work by means of a recording of their performance. The following is an example of this task; it is to measure the usability of a website game.

- Go to the website and enjoy your favourite game in the English section.

Each of these tasks consists of four sub-tasks which represent the key functional aspects of the selected website; i.e. they represent the main features of the website. Although they have been formed differently, the task goals are the same.

\section{Building the Questionnaire}

After the users finish their tasks they will be asked to rate their level of satisfaction with the website and task formalization on a scale of 1 to 7 , where 1 indicates 'highly unsatisfactory' and 7 indicates 'highly satisfactory'. This scale has been suggested to measure the levels of satisfaction felt by users truthfully following a test [Nielsen \& Loranger, 2006]. Also, they will be asked to write down their comments and thoughts in a feedback questionnaire, explaining any reactions that they observed during the test.

\section{User Characteristics}

Selecting and recruiting participants must be done carefully; the selection must reflect real users of the targeted websites because engaging inappropriate users will lead to incorrect results, thereby invalidating the experiments. Appropriate users will give confident results; the selection of these users will also encourage them to take part in the experiment [Dumas \& Redish, 1999]. In this experiment the majority of users of the targeted websites were students. They were divided into three groups consisting of 20 
students in each; each group was mixed in terms of sex, age, education and computer skills. These conditions should deliver reliable and accurate results; they may also play a significant role in the accuracy of the usability testing results. Of the 60 students, 40 were pre-university, and 20 were a mix of undergraduates and postgraduates. The users performing the specific tasks were called SC-Group, the users performing the guess tasks were called GC-Group, and the users performing the free tasks were called FR-Group.

\section{The Number of Users Participating in the Experiment}

As mentioned in the literature review, 20 users are required for testing each design of task and 'think-aloud' type. This means that 60 students are needed for each website, and, therefore, 180 users were required to conduct this experiment [Nielsen \& Loranger, 2006]. They were all introduced to the concept of the 'think-aloud' protocol and its three types, and how to perform the different task types.

\section{Test Environment}

The test environment was controlled; it was conducted in a quiet, child-friendly room. Also, we attempted to find out what our users regularly used, i.e. a familiar type of machine, browser and Internet connection speed, and set it up for them before the test.

Table 1: The actual environment for the usability testing experiment

\begin{tabular}{|l|l|}
\hline Test environment & $\begin{array}{l}\text { A small, quiet, } \\
\text { child-friendly room }\end{array}$ \\
\hline Type of machine & $\begin{array}{l}\text { Laptop (Intel, with } \\
\text { high speed and } \\
\text { Windows Vista) }\end{array}$ \\
\hline Browser & Internet Explorer 6 \\
\hline $\begin{array}{l}\text { Internet } \\
\text { connection }\end{array}$ & Reliable and fast \\
\hline $\begin{array}{l}\text { Additional } \\
\text { requirements }\end{array}$ & $\begin{array}{l}\text { A mouse for those } \\
\text { unfamiliar with a } \\
\text { laptop }\end{array}$ \\
\hline
\end{tabular}

\section{The Observer}

As mentioned above, usability testing requires an observer, and the researcher adopted this role in all the sessions, noting all the comments made by the users. $\mathrm{He}$ used a stopwatch to record the time spent by each user on each task, and an observation sheet to write down the behaviour of each user and the number of problems encountered.

\section{Severity Rating Assessment}

A severity assessment sheet was prepared in order to rank each usability problem on a scale of 0 to 4 in accordance with the literature review, and after listing all the usability problems they had encountered an evaluation was conducted for each user. This severity scale was used by Nielsen for prioritizing usability problems, as the severity sheets can then be used to compare the problems discovered by each group [Nielsen, 1994].

\section{Piloting the test}

There are two objectives in conducting a pilot study. The first one is to assess all the materials, including tasks, questionnaires and procedures, checking the language (to avoid any ambiguities) and ensuring that all the tasks have the same goal (although with different formulation). This objective was achieved by engaging independent expert evaluators. The second one is to assess the time needed for each test. This objective was achieved by engaging three users, each one performing one type of task. The users' behaviour was monitored and all the usability measures were assessed; the posttest questionnaires were then given to them by the observer (researcher) as they would be in the real testing. All these steps resulted in useful corrections and adjustments to the real test.

\section{Usability Measures Recorded}

In the experiment three usability attributes were considered, which were efficiency, effectiveness and satisfaction. These attributes were measured as time spent, task success and user satisfaction, respectively. 
- Time spent: the time spent on each task in seconds was recorded in order to measure the efficiency of the website.

- Task success rate: this was used to measure the effectiveness of the website. This was calculated using the following equation:

The success rate $=($ Number of tasks completed successfully) + (Number of tasks completed partly successfully $\left.\left({ }^{*} 0.5\right)\right)$ / the total number of tasks [Nielsen, 2001]

- User satisfaction: each user had to rate their satisfaction with the website after finishing the testing session, as indicated in Section 3.2.

Additional data were also collected:

- Number of errors: this refers to the number of errors made by a user when attempting to accomplish a task, such as visiting a wrong page.

- Usability problems encountered and their severity rate: this is the most important result as it can help the owner, developer and designer to improve their website. Once the problems were found they were assessed in terms of severity, as indicated in the literature review.

- Users' comments and feedback: this was collected at the end of each session; the users were asked to comment and to provide feedback with regard to their perceived usability of the website.

- Efficiency: one measure of Efficiency of Usability Evaluation Methods (UEMs) is "the ratio between the number of usability problems detected and the total time spent on the inspection process" [Fernandez et al., 2011]. Others can be deduced by dividing the average severity rating by the average time taken, as shown below [Tan et al., 2009]:

Efficiency $=\frac{\text { Average severity rating }}{\text { Average time spent }}$

[Tan et al., 2009]
- Thoroughness: this is perhaps the most attractive measure. It is defined as being an indication of the proportion of real problems found using a Usability Evaluation Method (UEM) to the total number of known real problems existing in the target design $\mathrm{z}$. It can be calculated by following formula:

Thoroughnes=

Number of real usability problems found

Total number of real usability problems

[Sears, 1997]

- Validity: this is the extent to which a usability evaluation method accurately identifies usability problems. It can be calculated by following formula:

Validity $=\frac{\text { Number of real usability problems found }}{\text { Number of issues identified as a usability problem }}$

[Sears, 1997]

- Effectiveness: this is defined as the ability of a usability evaluation method to identify usability problems related to the user interface of a specific system [Khajouei et al., 2011]. It can be calculated by the following formula:

Effectiveness $=\quad$ Thoroughness $\times$ Validity

[Sears, 1997]

\section{Data Analysis}

The previous sections describe and explore the objectives of the study, the methods used, the preparation of the experiment and, finally other usability testing measures. This section is a critical part of the experiment. It starts by exploring the users' demographic data, which includes nationality, gender and Web experience. It then examines the quantitative and qualitative data. Finally, the impact of the tasks and the 'think-aloud' types are assessed vis-à-vis on the three websites. Table 2 shows the mean values of the users' Web experience for all three groups. Approximately 4 years was the 
average for user experience across the three groups.

Table 2: Means for distribution of user Web experience

\begin{tabular}{|l|l|l|}
\hline Group & $\begin{array}{l}\text { Mean } \\
\text { (years) }\end{array}$ & $\begin{array}{l}\text { N (number of } \\
\text { users) }\end{array}$ \\
\hline $\begin{array}{l}\text { BBC } \\
\text { KS3bitesize }\end{array}$ & 3.7 & 60 \\
\hline Skoool & 4.1 & 60 \\
\hline $\begin{array}{l}\text { Academic } \\
\text { Earth }\end{array}$ & 4.4 & 60 \\
\hline Total & 4.1 & 180 \\
\hline
\end{tabular}

\section{Quantitative Analysis}

Quantitative data is an important part of usability data analysis. It provides a number of useful results, derived from various aspects that include success rate, error number, user satisfaction and time spent. The following sections explore and discuss the quantitative data collected in these experiments and reveal how each group performed with regard to usability measures.

\section{Success rate}

Table 3 shows the success rate for each group when using the three websites. The BBC KS3bitesize groups had the lowest overall success rate. For this website, FRGroup had the highest success rate of the three groups, which was expected because they performed tasks they were interested in and paid more attention to their tasks because they did not have to speak while doing them. They scored a 58\% success rate, which means that $58 \%$ of the tasks, on average, were completed successfully by the users. Although SC-Group discovered fewer usability problems than GC-group, they scored better than them. SC-Group members were observed performing tasks very successfully in the first stage, when they were asked to explore the website and familiarise themselves with it. They were more focused on accomplishing this task as they were expecting to encounter problems later. The actual tasks were limited to finding an answer and so they knew what they were looking for. Also, the constructive 'think-aloud' procedure helped them to clarify their tasks. This is perhaps the main difference between SC-Group and GC-group.

The Skoool groups ranked second in terms of success rate. They scored $62 \%$, which means that $62 \%$ of the tasks, on average, were completed successfully by the users. FRGroup again had the highest success rate, followed by SC-Group and GC-group respectively. It can be seen clearly that GCGroup were able to score more partially successful tasks than successful ones, as they attempted different ways and visited different pages to accomplish each task. Also, the concurrent 'think-aloud' procedure may have played a role in producing more failed tasks. This may be because this procedure distracted their attention, lowered their level of concentration and ultimately influenced their task performance. The Academic Earth groups had the highest success rate of the three website groups. These groups scoured 63\%, which means that $63 \%$ of the tasks, on average, were completed successfully by the users. SCGroup had the highest number of failed tasks of the three groups. This may be because they had doubts and were uncertain of their ability to guess and accomplish a task successfully. Overall, the FR-Group success rate was somewhat higher than those of SCGroup and GC-Group.

Despite these observations none of the three websites scored below 50\%, which is claimed by Nielsen to be the average for most websites, arguing that users generally fail to perform a task successfully the first time [Nielsen, 2001]. In terms of the group types, GC-Group achieved below 50\% in BBC KS3bitesize, and came close to this score with the other websites. This implies that the website's success rate can be described as acceptable and is in line with this study. 
Table 3: Success rate for the three groups across the three websites by three methods

\begin{tabular}{|c|c|c|c|c|c|c|c|}
\hline \multirow{2}{*}{$\begin{array}{l}\text { Type of } \\
\text { website }\end{array}$} & \multirow{2}{*}{$\begin{array}{l}\text { Group } \\
\text { Type }\end{array}$} & \multicolumn{5}{|c|}{ Task completion } & \multirow{2}{*}{$\begin{array}{l}\text { Mean success } \\
\text { rate for each } \\
\text { website }\end{array}$} \\
\hline & & $\begin{array}{l}\text { Successful } \\
\text { tasks }\end{array}$ & $\begin{array}{c}\text { Partially } \\
\text { successful } \\
\text { tasks }\end{array}$ & $\begin{array}{c}\text { Failed } \\
\text { tasks }\end{array}$ & $\begin{array}{l}\text { Number } \\
\text { of tasks }\end{array}$ & $\begin{array}{c}\text { Success } \\
\text { rate }\end{array}$ & \\
\hline \multirow{3}{*}{$\begin{array}{l}\text { BBC } \\
\text { KS3bitesize }\end{array}$} & $\begin{array}{l}\text { SC- } \\
\text { Group }\end{array}$ & 31 & 20 & 29 & 80 & $51 \%$ & \multirow{3}{*}{$52 \%$} \\
\hline & $\begin{array}{l}\text { GC- } \\
\text { Group }\end{array}$ & 27 & 19 & 34 & 80 & $46 \%$ & \\
\hline & $\begin{array}{l}\text { FR- } \\
\text { Group }\end{array}$ & 37 & 18 & 25 & 80 & $58 \%$ & \\
\hline \multirow{3}{*}{ Skoool } & $\begin{array}{l}\text { SC- } \\
\text { Group }\end{array}$ & 40 & 23 & 17 & 80 & $64 \%$ & \multirow{3}{*}{$62 \%$} \\
\hline & $\begin{array}{l}\text { GC- } \\
\text { Group }\end{array}$ & 33 & 24 & 23 & 80 & $56 \%$ & \\
\hline & $\begin{array}{l}\text { FR- } \\
\text { group }\end{array}$ & 48 & 23 & 9 & 80 & $66 \%$ & \\
\hline \multirow{4}{*}{$\begin{array}{l}\text { Academic } \\
\text { Earth }\end{array}$} & $\begin{array}{l}\text { SC- } \\
\text { Group }\end{array}$ & 38 & 21 & 30 & 80 & $61 \%$ & \multirow{3}{*}{$63 \%$} \\
\hline & $\begin{array}{l}\text { GC- } \\
\text { Group }\end{array}$ & 34 & 26 & 20 & 80 & $59 \%$ & \\
\hline & $\begin{array}{l}\text { FR- } \\
\text { Group }\end{array}$ & 41 & 27 & 12 & 80 & $68 \%$ & \\
\hline & & \multicolumn{5}{|c|}{ Average of the most websites [Nielsen 2001] } & $<50 \%$ \\
\hline
\end{tabular}

\section{The number of errors made}

Table 4 shows that the BBC KS3bitesize groups made more errors than the other website groups. FR-Group made fewer errors than the other groups, where an average of 1.5 errors were made by each user while he/she performed this type of task using 'think-aloud' protocol on the BBC KS3bitesize website, and 1.3 and 1.2 errors respectively on the Skoool and Academic Earth websites. GC-Group made the most errors. This was probably because this group did not have any guidelines or specific objective when performing the tasks. Also, the concurrent 'think-aloud' procedure may have had a negative impact, thus contributing to the number of errors made. Difficulties were also encountered because some of the navigation on the Skoool and Academic Earth websites took the users away from the website; also, there were too many windows and help links, and much of the documentation was not very visible. SCGroup came second with 1.7 errors per user, on average, in performing the specific task and the constructive 'think-aloud' procedure on the BBC KS3bitesize website; the average number of errors was 1.5 on both the Skoool and the Academic Earth websites. Thus, it can be seen that different types of task produce different results with regard to the number of errors made by users. 
Table 4: Error numbers for the three website groups by the three methods

\begin{tabular}{|l|c|c|c|c|c|c|c|c|c|}
\hline \multirow{2}{*}{ Website } & \multicolumn{3}{|c|}{ BBC KS3bitesize } & \multicolumn{3}{c|}{ Skoool } & \multicolumn{3}{c|}{ Academic Earth } \\
\cline { 2 - 10 } & $\begin{array}{l}\text { SC- } \\
\text { Grou } \\
\mathrm{p}\end{array}$ & $\begin{array}{l}\text { GC- } \\
\text { Group }\end{array}$ & $\begin{array}{l}\text { FR- } \\
\text { Group }\end{array}$ & $\begin{array}{l}\text { SC- } \\
\text { Group }\end{array}$ & $\begin{array}{l}\text { GC- } \\
\text { Group }\end{array}$ & $\begin{array}{l}\text { FR- } \\
\text { Group }\end{array}$ & $\begin{array}{l}\text { SC- } \\
\text { Group }\end{array}$ & $\begin{array}{l}\text { GC- } \\
\text { Group }\end{array}$ & $\begin{array}{l}\text { FR- } \\
\text { Group }\end{array}$ \\
\hline $\begin{array}{l}\text { No. of errors } \\
\text { made }\end{array}$ & 139 & 154 & 118 & 119 & 133 & 105 & 108 & 115 & 95 \\
\hline $\begin{array}{l}\text { Average } \\
\text { error } \\
\text { number per } \\
\text { user for each } \\
\text { task }\end{array}$ & 1.7 & 1.8 & 1.5 & 1.5 & 1.6 & 1.3 & 1.4 & 1.4 & 1.2 \\
\hline $\begin{array}{l}\text { Average } \\
\text { error } \\
\text { number per } \\
\text { user overall } \\
\text { tasks }\end{array}$ & 6.9 & 7.7 & 5.9 & 5.9 & 6.7 & 5.3 & 5.4 & 5.8 & 4.7 \\
\hline
\end{tabular}

\section{Number of usability problems discovered}

Table 5 shows the number of problems discovered by each group. It shows that GCGroup, in performing guessing tasks with the concurrent procedure, discovered $81 \%$ of the total usability problems discovered by all groups on the BBC KS3bitesize website, and $85 \%$ and $67 \%$ on the Skoool and Academic Earth websites respectively. SC-Group performed specific tasks using Co-Discovery Learning, uncovered only $56 \%$ of the total number of discovered usability problems on the BBC KS3bitesize website, and 39\% and $50 \%$ on the Skoool and Academic Earth websites respectively. The FR-Group found $31 \%$ of the total number of usability problems discovered by all groups on the BBC KS3bitesize website, and 31\% and 25\% on the Skoool and Academic Earth websites respectively. GC-Group performed better than SC-Group in discovering catastrophic, major, minor and cosmetic problems. However, FR-Group was better at discovering minor and cosmetic problems. The majority of the major problems discovered by GC-Group were mostly to do with navigation and structure. This may be because the users were not provided with guidelines telling them what they should do and where they should go; also, they had to speak out loud. On the other hand, SC-Group performed their tasks knowing what they were looking for, i.e. answering a question.

Table 5: Number of usability problems discovered by the three groups using three different methods

\begin{tabular}{|l|c|c|c|c|c|c|c|c|c|}
\hline \multirow{2}{*}{$\begin{array}{l}\text { Problem } \\
\text { type }\end{array}$} & \multicolumn{3}{|l|}{ BBC KS3bitesize } & \multicolumn{2}{l|}{ Skoool } & \multicolumn{2}{l|}{ Academic Earth } \\
\cline { 2 - 10 } & $\begin{array}{l}\text { SC- } \\
\text { Group }\end{array}$ & $\begin{array}{l}\text { GC- } \\
\text { Group }\end{array}$ & $\begin{array}{l}\text { FR- } \\
\text { Group }\end{array}$ & $\begin{array}{l}\text { SC- } \\
\text { Group }\end{array}$ & $\begin{array}{l}\text { GC- } \\
\text { Group }\end{array}$ & $\begin{array}{l}\text { FR- } \\
\text { Group }\end{array}$ & $\begin{array}{l}\text { SC- } \\
\text { Group }\end{array}$ & $\begin{array}{l}\text { GC- } \\
\text { Group }\end{array}$ & $\begin{array}{l}\text { FR- } \\
\text { Group }\end{array}$ \\
\hline $\begin{array}{l}\text { No. of } \\
\text { problems }\end{array}$ & 9 & 13 & 5 & 5 & 11 & 4 & 6 & 8 & 3 \\
\hline $\begin{array}{l}\% \text { of } \\
\text { discovered } \\
\text { problems }\end{array}$ & $56 \%$ & $81 \%$ & $31 \%$ & $39 \%$ & $85 \%$ & $31 \%$ & $50 \%$ & $67 \%$ & $25 \%$ \\
\hline Catastrophic & 0 & 0 & 0 & 1 & 1 & 0 & 0 & 0 & 0 \\
\hline Major & 1 & 2 & 1 & 1 & 3 & 0 & 2 & 3 & 1 \\
\hline Minor & 3 & 4 & 2 & 1 & 1 & 1 & 0 & 1 & 1 \\
\hline Cosmetic & 5 & 7 & 2 & 2 & 6 & 3 & 4 & 4 & 1 \\
\hline
\end{tabular}




\section{Time spent}

Table 6 shows the time spent by each user in performing their tasks, and whether or not they succeeded in carrying them out. GCGroup spent the longest time, longer than SCGroup and FR-Group, the timings being 310, 275 and 244 minutes respectively on the BBC KS3bitesize website. On the Skoool website, GC-Group spent the longest time 340 minutes; SC-Group and FR-Group spent 293 and 237 minutes respectively. On the Academic Earth website, again GC-Group spent the longest time, longer than SC-Group and FR-Group, the timings being 298, 267 and 239 minutes respectively. Again, this is probably due to the type of task and 'thinkaloud' procedure performed by the users. For example, GC-Group found themselves in problematic circumstances and having to speaking out loud whilst performing their tasks, which may have affected their concentration and attention. Also, they were trying to understand the situation they were in, and so had to spend time thinking about how to perform each task. FR-Group was free of such hindrances and so were more interested in, and concentrated on, accomplishing their tasks. SC-Group felt confident about how to accomplish their tasks and the constructive interaction procedure was helpful for this group; this is demonstrated in the success rate table (Table 3). The average time spent by each user in all website groups was longer than 3 minutes. Dumas and Redish (1999) asserted that the time spent by users on a website is less important than other factors such as success rate.

Table 6: Time spent by the three groups using the three methods

\begin{tabular}{|c|c|c|c|c|c|c|c|c|c|c|c|c|}
\hline \multirow{2}{*}{$\begin{array}{l}\text { Usability } \\
\text { measure }\end{array}$} & \multicolumn{3}{|c|}{ BBC KS3bitesize } & \multirow{2}{*}{$\begin{array}{l}\text { Total } \\
\text { time }\end{array}$} & \multicolumn{3}{|c|}{ Skoool } & \multirow{2}{*}{$\begin{array}{l}\text { Total } \\
\text { time }\end{array}$} & \multicolumn{3}{|c|}{ Academic Earth } & \multirow{2}{*}{$\begin{array}{l}\text { Total } \\
\text { time }\end{array}$} \\
\hline & $\begin{array}{l}\text { SC- } \\
\text { Group }\end{array}$ & $\begin{array}{l}\text { GC- } \\
\text { Group }\end{array}$ & \begin{tabular}{|l} 
FR- \\
Group
\end{tabular} & & $\begin{array}{l}\text { SC- } \\
\text { Group }\end{array}$ & $\begin{array}{l}\text { GC- } \\
\text { Group }\end{array}$ & $\begin{array}{l}\text { FR- } \\
\text { Group }\end{array}$ & & $\begin{array}{l}\text { SC- } \\
\text { Group }\end{array}$ & $\begin{array}{l}\text { GC- } \\
\text { Group }\end{array}$ & $\begin{array}{l}\text { FR- } \\
\text { Group }\end{array}$ & \\
\hline $\begin{array}{l}\text { Total time } \\
\text { spent by all } \\
\text { users (in } \\
\text { minutes) }\end{array}$ & 275 & 310 & 244 & 276 & 293 & 340 & 237 & 290 & 267 & 298 & 239 & 268 \\
\hline $\begin{array}{l}\text { Average } \\
\text { time per } \\
\text { user per } \\
\text { task (in } \\
\text { minutes) }\end{array}$ & 3.4 & 3.9 & 3.1 & 3.5 & 3.7 & 4.3 & 3 & 3.7 & 3.3 & 3.7 & 3 & 3.3 \\
\hline $\begin{array}{l}\text { Average } \\
\text { time per } \\
\text { user over } \\
\text { four tasks }\end{array}$ & 13.8 & 15.5 & 12.2 & 13.8 & 14.7 & 17 & 11.9 & 14.5 & 13.4 & 14.9 & 12 & 13.4 \\
\hline
\end{tabular}

\section{User Satisfaction}

Table 7 shows that the Academic Earth group were noticeably less satisfied than the BBC KS3bitesize and the Skoool groups. The FR-Group produced the highest scores, followed by SC-Group and GC-Group in overall evaluations. This indicates that there were certain factors influencing the users which impacted on their satisfaction score for the tested websites, as evidenced by the critical user comments relating to the design features of each website. These factors related to the various activities and 
functions, such as the games or the 'test and revise' facilities. Also, the users were influenced by the design of each website; a simple and attractive design encouraged and was enthused them. The best example is the BBC KS3bitesize website, which achieved the baseline score for user satisfaction of 4.7 , as determined by Nielsen and Loranger (2006) after they had tested 25 websites. Furthermore, it can be seen that FR-Group had the highest satisfaction score of the three website groups. This is probably because the users were given the freedom to perform the required tasks and the retrospective 'think-aloud' procedure was very helpful in achieving their high success rate. GC-Group, using the concurrent procedure had the lowest satisfaction score. This may reflect the fact that they were not given any clues or suggestions to help them complete their tasks, and having to speak out loud may have caused them a certain amount of frustration.

Table 7: User satisfaction scores of the three groups using the three methods

\begin{tabular}{|c|c|c|c|c|c|c|c|c|c|c|c|c|}
\hline \multirow{2}{*}{$\begin{array}{l}\text { Usability } \\
\text { measure }\end{array}$} & \multicolumn{3}{|c|}{ BBC KS3bitesize } & \multirow{2}{*}{$\begin{array}{l}\text { Average } \\
\text { score }\end{array}$} & \multicolumn{3}{|c|}{ Skoool } & \multirow{2}{*}{$\begin{array}{l}\text { Average } \\
\text { score }\end{array}$} & \multicolumn{3}{|c|}{ Academic Earth } & \multirow{2}{*}{$\begin{array}{l}\text { Average } \\
\text { score }\end{array}$} \\
\hline & $\begin{array}{l}\text { SC- } \\
\text { Group }\end{array}$ & $\begin{array}{l}\text { GC- } \\
\text { Group }\end{array}$ & $\begin{array}{l}\text { FR- } \\
\text { Group }\end{array}$ & & $\begin{array}{l}\text { SC- } \\
\text { Group }\end{array}$ & $\begin{array}{l}\text { GC- } \\
\text { Group }\end{array}$ & $\begin{array}{l}\text { FR- } \\
\text { Group }\end{array}$ & & $\begin{array}{l}\text { SC- } \\
\text { Group }\end{array}$ & $\begin{array}{l}\text { GC- } \\
\text { Group }\end{array}$ & $\begin{array}{l}\text { FR- } \\
\text { Group }\end{array}$ & \\
\hline $\begin{array}{l}\text { User } \\
\text { Satisfaction }\end{array}$ & 4.6 & 4.1 & 4.9 & $\overline{4.6}$ & 4.5 & 4.2 & 4.5 & 4.4 & 4 & 3.9 & 4.3 & 4.1 \\
\hline
\end{tabular}

\section{$>$ Usability Metrics}

It is noticeable in Table 8 that GC-group was more efficient in detecting usability problems and in the total time they spent on the inspection process on the three websites. Also, they were more thorough in discovering the real usability problems on the target websites. Furthermore, they achieved better validity in accurately identifying usability problems on the three websites. Finally, their ability to identify a usability problem that related to the user interface of a specific website was also higher. The SC-Group came second after GCgroup, and the FR-Group got the worst result from the three websites.

Table 8: Usability metric scores of the three groups using the three methods

\begin{tabular}{|l|l|c|l|c|c|c|}
\hline \multicolumn{1}{|c|}{ Additional Metric } & \multicolumn{2}{c|}{$\begin{array}{c}\text { BBC } \\
\text { KS3bitesize }\end{array}$} & \multicolumn{2}{c|}{ Skoool } & \multicolumn{2}{c|}{ Academic Earth } \\
\hline \multirow{2}{*}{$\begin{array}{l}\text { Efficiency by total } \\
\text { problems uncovered }\end{array}$} & SC-Group & 1 & SC-Group & 0.8 & SC-Group & 0.8 \\
\cline { 2 - 8 } & GC-Group & 1.4 & GC-Group & 1.24 & GC-Group & 1 \\
\cline { 2 - 8 } & FR-Group & 0.7 & FR-Group & 0.42 & FR-Group & 0.5 \\
\hline \multirow{4}{*}{ Thoroughness } & SC-Group & 0.4 & SC-Group & 0.5 & SC-Group & 0.3 \\
\cline { 2 - 8 } & GC-Group & 0.4 & GC-Group & 0.6 & GC-Group & 0.4 \\
\cline { 2 - 8 } & FR-Group & 0.2 & FR-Group & 0.5 & FR-Group & 0.4 \\
\hline \multirow{3}{*}{ Validity } & SC-Group & 0.4 & SC-Group & 0.5 & SC-Group & 0.3 \\
\cline { 2 - 7 } & GC-Group & 0.5 & GC-Group & 0.6 & GC-Group & 0.5 \\
\cline { 2 - 7 } & FR-Group & 0.6 & FR-Group & 0.3 & FR-Group & 0.6 \\
\hline & SC-Group & 0.2 & SC-Group & 0.4 & SC-Group & 0.1 \\
\cline { 2 - 7 } & GC-Group & 0.2 & GC-Group & 1 & GC-Group & 0.2 \\
\cline { 2 - 7 } & FR-Group & 0.1 & FR-Group & 0.2 & FR-Group & 0.2 \\
\hline
\end{tabular}

\section{Discussion and Findings}

This section examines the results of this experiment, highlighting the main findings and drawing out the lessons learned.
The main findings of this experiment are as follows:

1. This study proves that differences in the formulation of the tasks can affect the 
user experience and, therefore, the product evaluation of the end users. This means that the owners of websites or researchers should be careful to choose a task formulation that serves their goals by understanding the users' requirements. For example, the task formulation can indeed influence the results by revealing the different types of problems.

2. This study also proves that the 'thinkaloud' protocol can affect the way users think and behave during the session. For example, GS-Group, who performed the guess tasks with the concurrent 'thinkaloud' type of protocol, discovered more catastrophic, major, minor and cosmetic problems than the other two groups. However, SC-Group, who performed specific tasks with Co-Discovery Learning, discovered mostly major, minor and cosmetic problems. FR-Group, who performed free tasks with the retrospective 'think-aloud' procedure, revealed only minor and cosmetic problems. Thus, exploring the behaviour of users as they are engaged with a website and employing a variety of tasks can positively help in the development of a website.

3. From the observation of GS-Group, the concurrent 'think-aloud' type of evaluation appears to be the main reason for tasks not being completed because it leads to the dispersal of concentration of the users. This was manifested very obviously by the success of FR-Group when they performed their tasks silently. Consequently, this 'think-aloud' type should be avoided if the aim of the evaluation is to find all the usability problems in the selected product.

4. The task and type of 'think-aloud' protocol affected different measurements of usability, such as success rate and time spent. FR-Group achieved the highest success rate, made the fewest errors, had the highest level of user satisfaction and spent the least time. GC-Group, who used the concurrent 'think-aloud' procedure, left a number of tasks unfinished; they spent more time, made more errors and had lower levels of user satisfaction.
5. There is a relationship between user satisfaction and task type or 'thinkaloud' type; it is likely that if users make more errors while performing tasks, their satisfaction level will fall.

6. There is a strong positive correlation between the time spent and problems discovered (using Pearson Correlation), its significance being ( $p$-value $=0.02$ ), which means that when users spent more time they were able to discover more problems. Furthermore, there is also a strong positive correlation between the time spent and the number of errors made (using Pearson Correlation), the p- value being 0.03 .

7. In comparing the results of the testing methods described here with those of the traditional testing methods in AlRoobaea et al., (2013), the traditional user testing was better at discovering usability problems than the three combination methods. For example, the traditional testing of the BBC KS3bitesize website identified 16 problems, whereas SC Group, GC Group, and FR Group identified 9, 13, and 5 respectively. On Skoool website the traditional testing identified 13 problems, whereas SC Group, GC Group, and FR Group identified 5, 11, and 4 respectively. Finally, the traditional testing of Academic Earth website identified 12 problems, whereas SC Group, GC Group, and FR Group identified 6,8 , and 3 respectively. This leads to a recommendation that using traditional user testing without employing 'think-aloud' approaches, and using simple tasks, is a good formula for the discovery of any usability problems that may be present.

8. This study found (from the feedback questionnaire) that teenagers like websites that provide interactive features such as online tests, message boards for providing feedback, asking questions and playing games. All these features are present on the BBC KS3bitesize website and this could well be why this website achieved a high satisfaction rate. The low satisfaction rate achieved by the Academic Earth website might be related to the huge amount of information on each page, 
which confused the users during testing and impacted on their level of satisfaction, as teenagers do not like to read a great deal on a website or to have to look hard for links.

\section{Conclusion}

This paper investigated the impact on the results of usability testing in using three combinations of three tasks and three 'thinkaloud' approaches. These combinations are a specific task with 'constructive interaction think-aloud', 'guess task with concurrent think-aloud' and, finally, 'free task with retrospective think-aloud'. It has been shown that having different combinations of task design and 'think-aloud' approach can affect usability testing results by discovering different usability problems. This was demonstrated through the results of the assessment of the sites' usability obtained from the experiment in terms of the problems found. However, the study proves that there is no need to design complex task scenarios to discover more usability problems or to employ 'think-aloud' approaches. Designing suitable tasks based on the website features and understanding the users' experiences is quite sufficient to obtain good results. Further investigation on how different combinations of task design and think-aloud approaches can affect usability testing results is needed to confirm the findings of this research. In this regard, we propose to examine the impact of employing different combinations of 'thinkaloud' approaches and task designs; for example, employ the 'specific task' with the 'retrospective think-aloud' type or the 'guess task' with the 'constructive interaction thinkaloud' type, and also to employ these same combinations on different websites.

\section{Acknowledgements}

We thank the expert evaluators at the School of Education and Lifelong Learning and the School of Computing Sciences at the University of East Anglia (UEA), and the MSc and PhD students at both these schools, and also at the Saudi Norwich School (UK) and Thaghr School in Saudi Arabia. Thanks also go to the expert evaluators in Aviva company in the UK for their participation in this comparative study and in the mini-usability testing experiments.

\section{References}

Al-Badi, A., Michelle, O. Okam, Al Roobaea, R., and Mayhew, P. (2013), "Improving Usability of Social Networking Systems: A Case Study of LinkedIn," Journal of Internet Social Networking \& Virtual Communities, Vol. 2013 (2013), Article ID 889433, DOI: 10.5171/2013.889433.

Alghamdi,A., Al-Badi, A., Al Roobaea, R., and Mayhew, P. (2013). A Comparative Study of Synchronous and Asynchronous Remote Usability Testing Methods. International Review of Basic and Applied Sciences. Vol. 1 Issue.3.

Alrobai, A., AlRoobaea, R., Al-Badi, A., and Mayhew, P. (2012). Investigating the usability of e-catalogue systems: modified heuristics vs. user testing, Journal of Technology Research.

AlRoobaea, R., Al-Badi, A., and Mayhew P. (2013). A Framework for Generating Domain-Specific Heuristics for Evaluating Online Educational Websites. International Journal of Information Technology \& Computer Science, Volume 8, page 75 - 84.

AlRoobaea, R., Al-Badi, A., and Mayhew P. (2013). A Framework for Generating Domain-Specific Heuristics for Evaluating Online Educational Websites- Further Validation. International Journal of Information Technology \& Computer Science, volume 8, page 97 - 105.

AlRoobaea, R., Al-Badi, A., and Mayhew, P. (2013). Generating a Domain Specific Inspection Evaluation Method through an Adaptive Framework: A Comparative Study on Educational Websites. International Journal of Human Computer Interaction (IJHCI), 4(2), 88.

AlRoobaea, R., Al-Badi, A., and Mayhew, P. (2013). Generating a Domain Specific Inspection Evaluation Method through an Adaptive Framework. International Journal of Advanced Computer Science and Applications, Vol.4 No.6. 
Alshamari, M. and Mayhew, P. (2008). Task design: Its impact on usability testing. In Internet and Web Applications and Services, 2008, ICIW'08. Third International

Conference on, pages 583-589. IEEE.

AlShamari, M., and Mayhew, P. (2010). Task Formulation in Usability Testing (Doctoral dissertation, University of East Anglia).

Dale, O., Drivenes, T., Tollefsen, M., and Reinertsen, A. (2012). User Testing of Social Media-Methodological Considerations. In Computers Helping People with Special Needs (pp. 573-580). Springer Berlin Heidelberg.

Dix, A. (2004). Human-computer interaction. Prentice Hall.

Dumas, J. and Redish, J. (1999). A practical guide to usability testing, Lives of Great Explorers Series, Intellect Books, Portland.

Ebling, M. and John, B. (2000). On the contributions of different empirical data in usability testing. In Proceedings of the $3^{\text {rd }}$ Conference on Designing Interactive Systems: processes, practices, methods and techniques, pages 289-296. ACM.

Fang, X., and Holsapple, C. (2011). Impacts of navigation structure, task complexity, and users' domain knowledge on Web site usability-an empirical study. Information Systems Frontiers, 13(4), 453-469.

Feng, J., Lazar, J., Kumin, L. and Ozok, A. (2010). Computer usage by children with Down's syndrome: Challenges and future research. ACM Transactions on Accessible Computing (TACCESS), 2(3): 13.

Fernandez, A., Insfran, E. and Abrahão, S., (2011), Usability evaluation methods for the web: A systematic mapping study, Information and Software Technology.

Garrett, J. (2010). The elements of user experience: user-centered design for the Web and beyond. New Riders Pub.

Hornbaek, K. (2006). Current practice in measuring usability: Challenges to usability studies and research. International journal of human-computer studies, 64(2): 79-102.
ISO ISO. 9241-11: 1998 ergonomic requirements for office work with visual display terminals (vdts) - Part 11: guidance on usability. Geneve, CH: ISO, 1998.

Jeffries, R., Miller, J.R., Wharton, C. and Uyeda, K.M. (1991). User interface evaluation in the real world: A comparison of four techniques. Proceedings of ACMCHI'91, pp. 119-124. New York: ACM Press.

Khajouei, R., Hasman, A. and Jaspers, M., (2011), Determination of the effectiveness of two methods for usability evaluation using a CPOE medication ordering system, International Journal of Medical Informatics, vol. 80 (5), pp. 341-350.

Krahmer, E. and Ummelen, N. (2004). Thinking about thinking aloud: A comparison of two verbal protocols for usability testing. Professional Communication, IEEE Transactions on, 47(2): 105-117.

Law, L. and Hvannberg, E. (2002), complementarily and convergence of heuristic evaluation and usability test: a case study of universal brokerage platform. In Proceedings of the Second Nordic Conference on Human-Computer Interaction, pages 71-80, ACM.

Lewis, C. and Polson, P. (1992). Cognitive walkthroughs: A method for theory-based evaluation of user interfaces. Paper presented at the Tutorial at the CHI'92 Conference on Human Factors in Computing Systems, Monterey, CA.

Liljegren, E., (2006), Usability in a medical technology context assessment of methods for usability evaluation of medical equipment, International Journal of Industrial Ergonomics, vol. 36 (4), pp. 345-352.

Lindgaard, G. and Chattratichart, J. (2007). Usability testing: what have we overlooked? In Proceedings of the SIGCHI Conference on Human Factors in Computing Systems, pages 1415-1424. ACM.

Manzari, L., and Trinidad-Christensen, J. (2013). User-centered design of a web site for library and information science students: 
Heuristic evaluation and usability testing. Information technology and libraries, 25(3), 163-169.

Molich, R., Ede, M., Kaasgaard, K. and Karyukin, B. (2004). Comparative usability evaluation. Behaviour \& Information Technology, 23(1):65-74.

Nielsen, J. \& Molich, R. (1990). Heuristic evaluation of user interfaces. Proceedings of the ACM CHI'90 Conference on Human Factors in Computing Systems, 249-256. New York: ACM.

Nielsen, J. (1994). Usability engineering. Morgan Kaufmann.

Nielsen, J. (2000). Why you only need to test with 5 users. Test 9 (September 23).

Nielsen, J. (2001). Success rate: The simplest usability metric, available at:

[http://www.useit.com/alertbox/20010218. html], accessed on $11 / 6 / 2012$

Nielsen, J. (2006). Quantitative studies: How many users to test. Alertbox, June, 26:2006.

Nielsen, J. (2009). Authentic behavior in user testing.

Nielsen, J. and Loranger, H. (2006), Prioritizing web usability, New Riders Press, Thousand Oaks, CA, USA.

Oztekin, A., Konga, Z., and Uysal, O. (2010). UseLearn: A novel checklist and usability evaluation method for eLearning systems by criticality metric analysis. International Journal of Industrial Ergonomics, 40(4): 455469, 2010.

Rubin, J. and Chisnell, D. (2008). Handbook of Usability Testing: How to Plan, Design and Conduct Effective Tests. Wiley India Pvt. Ltd.

Sauro, J. and Kindlund, E. (2005). A method to standardize usability metrics into a single score. In Proceedings of the SIGCHI Conference on Human Factors in Computing Systems, pages 401-409. ACM.

Sears, A., (1997), Heuristic walkthroughs: Finding the problems without the noise,
International Journal of Human-Computer Interaction, vol. 9 (3), pp. 213-234.

Tan, W., Liu, D. and Bishu, R. (2009). Web evaluation: Heuristic evaluation vs. user testing. International Journal of Industrial Ergonomics, 39(4): 621-627.

Tullis, T. and Albert, W. (2008). Measuring the User Experience: Collecting, Analyzing, and Presenting. Morgan Kaufmann, Burlington.

Tullis, T., Fleischman, S., McNulty, M., Cianchette, C. and Bergel, M. (2002). An empirical comparison of lab and remote usability testing of web sites. In Usability Professionals Association Conference. UPA.

Van den Haak, M., de Jong, M. and Schellens, P. (2004). Employing think-aloud protocols and constructive interaction to test the usability of online library catalogues: a methodological comparison. Interacting with computers, 16(6): 1153-1170. 